\title{
Análisis factorial comprobatorio de la herramienta SERVQUAL en alojamiento. Amazonia Ecuador
}

\section{Verifying factor analysis of the SERVQUAL tool in accommodation. Amazon Ecuador}

Gary Patricio Rivadeneyra Olalla. ${ }^{1}$, Edison Eduardo Ruiz Mármol. ${ }^{2}$, Carlos Anibal Manosalvas Vaca. ${ }^{3} \&$ Álvaro Santiago Toalombo Díaz. ${ }^{4}$

\begin{abstract}
The present research topic "Verifying factor analysis of the SERVQUAL tool in accommodation. Amazonia Ecuador" responds to the growth of the tourist accommodation offer that in recent years has been concentrated in the urban area of the canton in which 108 establishments are registered in the different categories that offer their services without having a real knowledge of the perception and expectations of its users. The objective of the work was to evaluate the quality of the services offered by the 3-star lodging establishments of the Urbana de Tena parish. It was developed as a qualitative investigation for the compilation of previous information of the investigation variables and quantitative for the processing and analysis of the data obtained in the field investigation. Based on an analysis of the level of professionalization of the workers of the accommodation establishments, a compilation is made on the quality of the services provided through the SERVQUAL model, using the survey as a technique, applied to a randomly selected sample. Finally, they used statistics such as Cronbach's alpha and confirmatory analysis that determined the factors that influence the quality of the services provided. When applying the SERVQUAL model to accommodation establishments in the city of Tena, it was found that expectations, measured by tangible aspects, reliability, response capacity, security and empathy are considered important for the client, since the average score obtained from the survey applied to these was located in the range of 6.01 and 6.78 , which corresponds to a high expectation, and the

\footnotetext{
${ }^{1}$ Universidad Estatal Amazónica, Centro de Postgrados, Puyo-Ecuador, gp.rivadeneyrao@uea.edu.ec

${ }^{3}$ Universidad Estatal Amazónica, Facultad Ciencias de la Vida, Puyo-Ecuador, cmanosalvas@uea.edu.ec

${ }^{4}$ Universidad Estatal Amazónica, Centro de Postgrados, Puyo-Ecuador, as.toalombod@uea.edu.ec
}

${ }^{2}$ Universidad Estatal Amazónica, Centro de Postgrados, Puyo-Ecuador, eruiz@uea.edu.ec
\end{abstract}


confirmatory factor analysis determined that from the SERVQUAL questionnaire that 2 items do not meet the individual reliability $>0,50$

Keywords: Satisfaction, Quality, SERVQUAL, Factor analysis, Tourist offer.

\section{Resumen}

El presente tema de investigación "Análisis factorial comprobatorio de la herramienta SERVQUAL en alojamiento. Amazonia Ecuador" responde al crecimiento de la oferta turística de alojamiento que en los últimos años se ha concentrado en el sector urbano donde se registran 108 establecimientos de alojamiento en las diferentes categorías que ofertan sus servicios sin tener un conocimiento real de la percepción y expectativas que tienen sus usuarios. El objetivo del trabajo fue establecerla fiabilidad de los indicadores SERVQUAL de la calidad de los servicios ofertados por los establecimientos de hospedaje de la ciudad de Tena. Se desarrolló como una investigación cualitativa para la recopilación de información previa de las variables de investigación y cuantitativa para el procesamiento y análisis de los datos obtenidos en la investigación de campo. Se realiza una recopilación sobre la calidad de los servicios prestados a través del modelo SERVQUAL, empleando como técnica la encuesta, aplicada a una muestra seleccionada de manera aleatoria. Finalmente, se emplearon herramientas estadísticas como el alfa de Cronbach y análisis confirmatorio que determinó los factores que influyen en la calidad de los servicios prestados. Al aplicar el modelo SERVQUAL a los establecimientos de alojamiento de la ciudad de Tena se encontró que las expectativas, medidas por los aspectos tangibles, fiabilidad, capacidad de respuesta, seguridad y empatía son considerados importantes para el cliente, ya que el puntaje medio obtenido de la encuesta aplicada a estos se ubicó en el rango de 6.01 y 6.78, que corresponde a un alta expectativa y el análisis Factorial confirmatorio determinó que del cuestionario SERVQUAL que 2 ítems no cumplen con la fiabilidad individual >0,50

Palabras claves: Análisis factorial, Calidad, Oferta turística, Satisfacción, SERVQUAL.

\section{Introducción}

Ecuador es un país que dispone de una flora, fauna y posición geográfica privilegiadas que lo hacen disponer de una serie de maravillas naturales, indescriptibles, entre ellas la Amazonia, compartido con países cercanos como Brasil, Perú, Bolivia, el cual representa el bosque tropical más extenso del mundo, altamente reconocido por la biodiversidad que alberga, tales como el representativo rio amazona, ciudades ecológicas, arquitectura del periodo colonial, y un sinnúmero de espacios naturales, explorados y por explorar. Estas características hacen del lugar un espacio de particular atractivo turístico tanto para el 
visitante nacional como internacional, no obstante, pareciera continuar presentando distintas oportunidades de mejora en cuanto a la calidad del servicio ofertado por los operadores turísticos y establecimientos de alojamiento de la zona (Barreiro, 2015).

El hospedaje o alojamiento representa uno de los aspectos que inciden en la calidad de los servicios turísticos, ya que puede disponer de un lugar paradisiaco, flora y fauna encantadores, pero es el servicio ofrecido el que marca la pauta para que el turista realice su elección en cuanto a dónde hospedarse o cual producto escoger. Una de las herramientas para analizar la calidad del servicio es a través de la metodología SERVQUAL, empleando esta metodología es posible conocer las expectativas de los usuarios (MINTUR, 2018).

Al aplicar el modelo SERVQUAL es posible conocer las expectativas del cliente sobre un servicio en particular y la percepción o creencia que se forman los directivos de la empresa sobre lo que espera el consumidor de ese servicio, la percepción de los directivos de la empresa y las especificaciones o normas de calidad del servicio, las especificaciones o normas de calidad del servicio y la prestación del mismo (el servicio entregado), la prestación del servicio (servicio entregado) y lo que es comunicado acerca del servicio a los clientes (comunicación externa) y 1 servicio entregado y el servicio percibido, es decir, la calidad en el servicio (Crespo, 2019).

Las evaluaciones de calidad de servicio, se ubican dentro de las mejores prácticas en la gerencia de todos los proyectos, incluyendo el servicio de hospedaje de servicios turísticos, la calidad remite a uno de atributos o conjunto de cualidades características de algo, que propicia juzgar su valor; pero en el ámbito de negocios se ha definido como el cumplimiento de las especificaciones o lograr por adelantado tomando en cuenta lo que ocurrirá, sin haber efectuado la situación (Morales \& Fernández, 2019).

La calidad, también es definida como el resultado de combinaciones adecuadas de muchos parámetros tanto empíricos como no tangibles. Dicho de otra manera, representa un valor que denota los productos y servicios a ofertarse, en consecuencia, la aceptación en el consumidor puede verse incrementada (Salazar \& Cabrera, 2016).

En el Ecuador una de las normalizaciones y estándares de calidad para medir la calidad del servicio de alojamiento fue diseñado por la entidad QUALITUR, corporación de derecho 
privado que presenta como finalidad elevar la calidad en la prestación de servicios de alojamiento, mediante un sistema de certificación de competencias laborales con estudios e investigación, capacitación, normalización y evaluación para obtener la certificación (Guamangallo, Masapanta, \& Nasimba, 2019). por ello menciona doce normas para el sector alojamiento: encargado de reservas, recepcionista, ama de llaves, botones, camarera de pisos, servicio personalizado de información turística, encargado de mantenimiento, recepcionista polivalente, jefe de recepción, coordinador de eventos, auditor nocturno y administrador de empresas de alojamiento (Qualitur, 2020).

Los criterios presentados por QUALITUR para medir los parámetros de calidad hotelera se sustenta en los principios de capacitación de los empleados, sin embargo, existen otros estándares como es el caso de la propuesta diseñada por el propio ministerio del turismo, para evaluar la gestión y prestación de servicios, la cual tuvo lugar mediante la aplicación de encuestas de calidad de servicio dirigida a los usuarios (Qualitur, 2020).

Suministrar servicios de calidad, representan un aspecto fundamental, cuando se tiene por objetivo alcanzar la fidelidad de una proporción significativa de clientes. De allí surge la importancia de efectuar mediciones acerca de la calidad, actividad permite definir objetivos claros, determinar problemas y analizar las situaciones antes y después que se efectúan los cambios, adicionalmente, medir la satisfacción del consumidor, la cual puede ser cuantificada como la diferencia entre las expectativas y sus percepciones (Arciniegas \& Mejías, 2017). De acuerdo a este planteamiento, las empresas prestadoras de servicios se encuentran en una permanente búsqueda de lealtad de sus clientes, lo que se obtiene suministrando servicio de calidad, lo suficientemente competitivos. Las organizaciones que logran la adhesión de los clientes y su lealtad son expertas en comprender y responder a sus expectativas e, incluso, logran superar las necesidades del consumidor (Quispe \& Ayavarí, 2016).

El presente artículo aborda el tema de investigación "Análisis factorial comprobatorio de la herramienta SERVQUAL en alojamiento. Amazonia Ecuador". Modelo desde el cual, se consideran cinco dimensiones: fiabilidad, tangibilidad, capacidad de respuesta, confianza y empatía, las cuales consolidan las expectativas de los consumidores en relación con el servicio que reciben. En el presente caso se aplica el modelo debido al crecimiento de la oferta turística de alojamiento que durante los últimos años se ha concentrado en el cantón 
donde que se registran 108 establecimientos hoteleros en las diferentes categorías, los cuales ofertan sus servicios sin tener un conocimiento preciso acerca de la percepción y expectativas que presentan sus usuarios. Zeithaml, (2000) creador del modelo de SERVQUAL, proponen que los principales influyentes en la creación de las expectativas son la transmisión boca a boca por parte de los usuarios, las necesidades particulares que quiere satisfacer cada cliente, las experiencias precedentes que puede haber tenido acerca del servicio, y la comunicación corporativa que realiza la empresa.

El estudio presenta el objetivo de evaluar la calidad de los servicios ofertados por los establecimientos de alojamiento del Cantón Tena. Para alcanzar el fin se ejecutó un tipo de investigación cualitativa en la fase de recopilación de información previa de las variables de investigación y cuantitativa para el procesamiento y análisis de los datos obtenidos en la investigación de campo. Se realiza una recopilación sobre la calidad de los servicios prestados a través del modelo SERVQUAL empleando como técnica encuestas a una muestra seleccionada de manera aleatoria. Finalmente, se establece una prueba estadística que determina los factores que influyen en los 22 ítems del modelo SERVQUAL.

\section{Metodologia.}

\section{Característica generales del lugar}

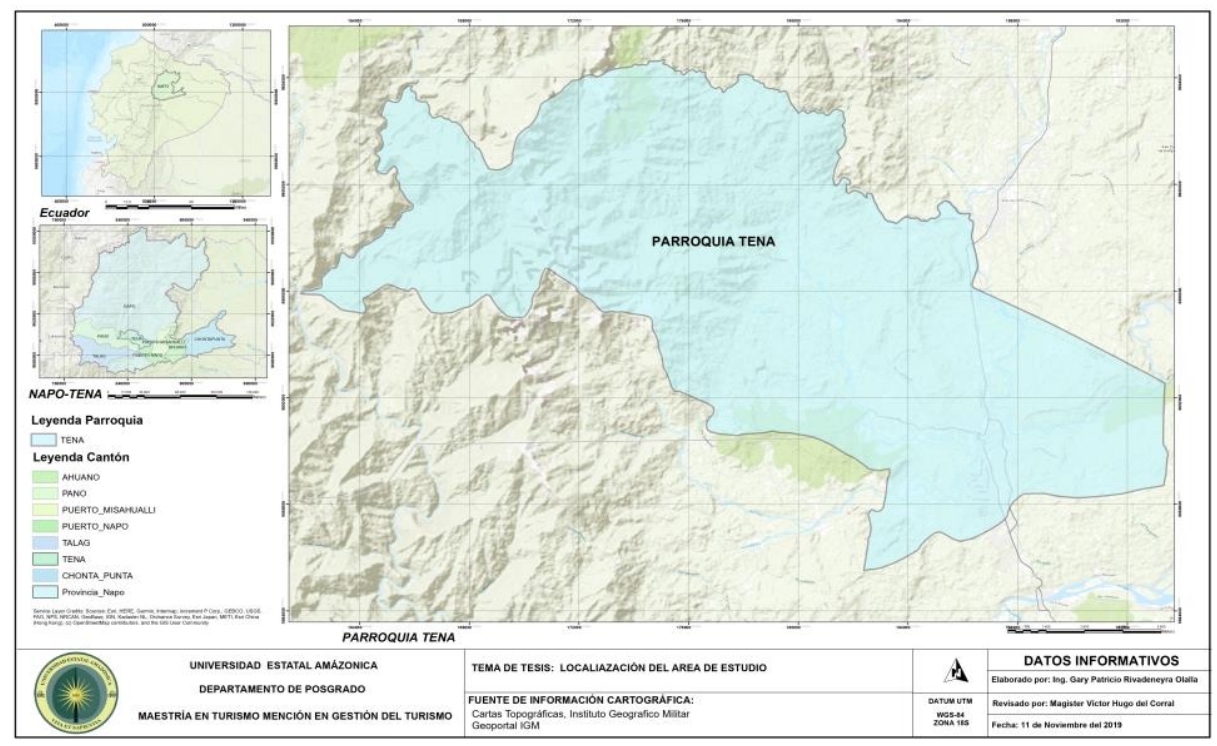

Gráfico 1 Localización geográfica de la parroquia Tena Fuente: GAD Municipal (2020) 
El estudio se ubica en la Parroquia Urbana del Cantón Tena, Provincia de Napo. Esta Parroquia se asienta en la unión de los Ríos Pano y Tena que dan vida al Rio Napo principal afluente del Rio Amazonas, la Parroquia limita al Norte con el Cantón Tena, al sur con el Cantón Carlos Julio Arosemena Tola, al este con la parroquia de Puerto Misahuallí y al oeste con la Parroquia del Talag. Las coordenadas se corresponden con 059'20' ' S77 ${ }^{\circ} 48^{\prime} 57^{\circ}$. La superficie total abarca $3.904 \mathrm{Km}$. La población alcanza una cantidad aproximada de 60.880 habitantes, quienes se desempeñan, principalmente en la agricultura, comercio y turismo (GAD Municipal de Tena, 2020).

El cantón Tena se corresponde con una localidad territorial subnacional, perteneciente a la provincia de Napo, ubicada por encima del valle del río Misahuallí y a una altitud de 510 metros sobre el nivel del mar, presenta un clima, promedio de 25 grados centígrados, caracterizado por ser cálido húmedo, ocasionado por la cercanía con la selva amazónica, presenta una humedad entre el 90 y 100\%. Topográficamente la ciudad se encuentra dividida en barrios, dentro de una misma parroquia (GAD Municipal de Tena, 2020).

\section{Tipo de investigación}

\section{Nivel de investigación}

El nivel de la investigación efectuada se corresponde con el descriptivo, lo que permitió dar cuenta del comportamiento de las variables de estudio, registrar y analizar los datos en profundidad, identificando las características del sector hotelero en la parroquia urbana Tena (Arias, El Proyecto de Investigación, 2016). Para analizar con detenimiento la información recabada se empleó el método teórico lógico hipotético deductivo por la necesidad de generar conclusiones particulares a través de datos obtenidos y desarrollarlos con el modelo SERVQUAL el cual mide la satisfacción del cliente en función de fiabilidad, sensibilidad, seguridad, empatía, y elementos tangibles (Hernández-Sampieri, et al., 2014, p. 94).

Por otro lado, se espera estimar el grado de relación entre las propiedades de los ítems y los compontes del modelo, SERVQUAL, mismo que evalúa dos etapas cruciales dentro del servicio: la primera la realiza antes de la entrega del servicio, expectativas, y la segunda después de haber consumido el servicio, percepción; mediciones que se efectúan basado en 
una escala de 5 puntos pertenecientes a las dimensiones ya mostradas con anterioridad con 22 ítems a analizarse; es decir, se tiene como finalidad conocer si efectivamente los ítems miden lo que pretenden medir y la calidad de dicha relación (Hernández-Sampieri, et al., 2014, p. 93).

\section{Tipo de la investigación}

El tipo de investigación al cual se alineó el presente trabajo fue cuantitativo, ya que permitió la recopilación de información para distinguir los factores de medición de calidad de la herramienta SERVQUAL por medio del análisis factorial comprobatorio, aplicado en el servicio de alojamiento en la ciudad de Tena, de forma que los datos obtenidos se convirtieron en unidades estadísticas, las cuales permitieron el análisis de las frecuencias en las que se encuentra la variable identificada en la presente investigación, obteniendo información del análisis factorial comprobatorio de los factores de medición de calidad que establece el modelo seleccionado (Hernández-Sampieri, et al., 2014, p. 58).

\section{Diseño de la investigación}

La presente investigación utiliza un diseño no experimental; en este sentido, se establece que el estudio es sistemático y empírico. La variable independiente no es controlada por el investigador y las inferencias sobre los indicadores de esta se realizan sin intervención o influencia directa, y dichas relaciones se observan tal como se han dado en su contexto natural (Hernández-Sampieri, et al., 2014, p. 153), asociado a discriminar los factores de medición de calidad de la herramienta SERVQUAL por medio del análisis factorial comprobatorio, aplicado en el servicio de alojamiento en la ciudad de Tena.

\section{Alcance de la investigación}

El alcance de la investigación se ubica en el nivel descriptivo, ya que permitió realizar un análisis factorial comprobatorio de la herramienta SERVQUAL en alojamiento. Amazonia Ecuador, en un momento especifico, es decir, durante la ejecución de la investigación, y dicho proceso no implica el seguimiento al comportamiento del mismo en otros tiempos o a más largo plazo (Hernández-Sampieri, et al., 2014, p. 120). 
El análisis confirmatorio implicó realizar el análisis de fiabilidad del instrumento aplicado y obtener el coeficiente de alfa de Cronbach global. Se obtuvo la fiabilidad interna de las dimensiones que conforman el modelo SERVQUAL, es decir, aspectos tangibles, fiabilidad, capacidad de respuesta, seguridad y empatía, verificando con ello la consistencia interna de los datos que fueron sometidos a rigurosos análisis estadísticos como es la prueba de alfa de Crombach, a través del cual se identificó la fiabilidad de las puntuaciones obtenidas para los ítems que conforman cada dimensión.

\section{Población y Muestra}

\section{Población}

Arias (2012) propone que la población de una investigación representa un conjunto que puede ser finito o infinito de todos los elementos con las mismas características. En el presente estudio la población la constituyeron los turistas que se hospedaron en los 108 establecimientos de alojamiento registrados en el Cantón Tena, tomando en cuenta la ocupación promedio del 6,62 \% (ocupación promedio del primer semestre del año 2019) durante el primer semestre del año 2020, en sus diferentes categorías, localizados en la parroquia y Cantón estudiado.

\section{Muestra:}

La muestra se corresponde con un subgrupo de la población, tal como lo señala Ventura (2017), en el caso del presente estudio correspondió con 121 personas, miembros de los distintos establecimientos de alojamiento localizados en la parroquia, quienes manifestaron su disposición de participar en el estudio realizado.

Tabla 1 Muestra

\begin{tabular}{lll}
\hline Sexo & FRECUENCIA & PORCENTAJE \\
\hline Femenino & $\mathbf{6 1}$ & $\mathbf{5 0 \%}$ \\
Masculino & $\mathbf{6 1}$ & $\mathbf{5 0 \%}$ \\
TOTAL & $\mathbf{1 2 2}$ & $\mathbf{1 0 0 \%}$ \\
\hline
\end{tabular}

Tabla 1. Elaborado por El investigador 


\section{Criterios de inclusión y exclusión}

Tabla 2 Criterios de inclusión y exclusión

INCLUSIÓN $\quad$ EXCLUSIÓN

Ser mayor de edad

Laboralmente activo en un alojamiento

Expresar voluntad de participar en el estudio
Ser menor de edad

Laboralmente inactivo o trabajar en otra rama

Expresar voluntad de no participar en el estudio

Tabla 2 Elaborado por El investigador

\section{Resultados.}

Tabla 3: Coeficiente Alfa de Cronbach global y para los componentes del modelo SERVQUAL

\begin{tabular}{lc}
\hline SERVQUAL & Alfa de Cronbach \\
\hline Total & 0,966 \\
Componentes & \\
Dimensión Aspecto Tangibles & 0,912 \\
Dimensión Fiabilidad & 0,922 \\
Dimensión Capacidad de Respuesta & 0,814 \\
Dimensión Seguridad & 0,929 \\
Dimensión Empatía & 0,933 \\
\hline
\end{tabular}

Fuente: Elaboración propia

Los resultados del coeficiente de alfa de Cronbach global obtenido fue de 0.966, considerado aceptable de acuerdo a la literatura, en cuanto a la consistencia interna de los componentes del modelo SERVQUAL se observa que oscila entre 0.912 y 0.933 . Se obtuvo la fiabilidad interna de las dimensiones que conforma el modelo SERVQUAL, es decir, aspectos tangibles (0.912), fiabilidad (0.922), capacidad de respuesta (0.814), seguridad (0.929) y empatía (0.933), verificando con ello que el coeficiente de consistencia interna es excelente para 4 dimensiones, ya que se obtuvo valores superiores a 0,90; mientras que el coeficiente de la 
dimensión capacidad de respuesta tiene un nivel consistencia bueno ya que el valor obtenido es mayor a 0,80 .

Una vez especificado el modelo SERVQUAL se consideró para el análisis 22 ítems y 3 dimensiones o variables no observables, agrupadas de la siguiente manera: para la Dimensión 1 correspondiente a Tangibilidad (T) se consideró los ítems 1,2,3 y 4; para la dimensión 2 que es Fiabilidad y Capacidad de Respuesta (F \& R)los ítems 5,6, 7, 8, 9, 10, 11, 12 y 13; finalmente para la tercera dimensión denominada Seguridad y Empatía (S y E) se conforma por los ítems 14, 15, 16, 17, 18, 19, 20, 21 y 22. Por ende como se visualiza en la Figura 1 no se eliminó ninguno de los ítems, puesto que todos explicaban el modelo y cumplieron con las especificaciones (fiabilidad individual >0,50).

Los resultados obtenidos evidencian que las cargas factoriales estandarizadas presentan valores superiores a 0,70, además, la probabilidad asociada al estadístico de prueba es significativo $(* * *)$ ya que todos los valores son inferiores a 0,05 . Por otro lado, la fiabilidad individual (R2) de cada ítem es igual o superior a 0,50, que es considerado aceptable. (Ver Tabla 5)

Tabla $\mathbf{N}^{\circ} 4$ Análisis Factorial Confirmatorio del modelo propuesto

\begin{tabular}{lllll}
\hline & & & Estimate & R2 \\
\hline Tangibilidad & $<---$ & T & 0,707 & 0,500 \\
Fiabilidad y Capacidad de Respuesta & $<---$ & F \& R & 0,735 & 0,540 \\
Seguridad y Empatía & $<--$ & T & 0,751 & 0,564 \\
P4 & $<---$ & T & 0,922 & 0,850 \\
P3 & $<---$ & T & 0,869 & 0,755 \\
P2 & $<---$ & T & 0,835 & 0,697 \\
P1 & $<---$ & T & 0,718 & 0,516 \\
P9 & $<---$ & F \& R & 0,675 & 0,456 \\
P8 & $<---$ & F \& R & 0,896 & 0,803 \\
\hline
\end{tabular}




\begin{tabular}{lcccc}
\hline P7 & $<---$ & F \& R & 0,908 & 0,824 \\
P6 & $<---$ & F \& R & 0,836 & 0,699 \\
P5 & $<---$ & F \& R & 0,832 & 0,692 \\
P11 & $<---$ & S \& E & 0,863 & 0,745 \\
P16 & $<---$ & S \& E & 0,864 & 0,746 \\
P15 & $<---$ & S \& E & 0,820 & 0,672 \\
P14 & $<---$ & S \& E & 0,857 & 0,734 \\
P10 & $<---$ & F \& R & 0,719 & 0,517 \\
P11 & $<---$ & F \& R & 0,846 & 0,716 \\
P12 & $<---$ & F \& R & 0,840 & 0,706 \\
P13 & $<---$ & F \& R & 0,336 & 0,513 \\
P18 & $<---$ & S \& E & 0,849 & 0,721 \\
P19 & $<---$ & S \& E & 0,803 & 0,645 \\
P20 & $-<$ & S \& E & 0,820 & 0,672 \\
P21 & & S \& E & 0,852 & 0,726 \\
P22 & & 0,734 & 0,539 \\
\hline
\end{tabular}

Fuente: Elaboración propia

\section{Validez}

En la Tabla 6 y Figura 1 se observan los resultados del análisis factorial confirmatorio (AFC) del modelo SERVQUAL aplicado a los clientes. Los parámetros estimados se obtuvieron a través del software estadístico Amos.

Se evidencia que las cargas factoriales estandarizadas presentan valores superiores a 0.70 , además, la probabilidad asociada al estadístico de prueba es significativo $(* * *)$ ya que todos los valores son inferiores a 0,05. Las correlaciones cuadradas múltiples (R2) de cada componente es igual o superior a 0,50 . 
Tabla $\mathbf{N}^{\circ} 5$ Análisis factorial confirmatorio de segundo orden para la muestra evaluada

\section{Componentes}

Correlaciones múltiples cuadradas

Tangibilidad $0.707(* * *)$ 0.501

Fiabilidad y Capacidad de Respuesta $0.735(* * *)$ 0.540

Seguridad y Empatía

$0.751(* * *)$

0.564

\section{Fuente: Elaboración propia}

Los resultados del análisis factorial confirmatorio (AFC) del modelo SERVQUAL aplicado a los clientes. Los parámetros estimados se obtuvieron a través del software estadístico Amos. Se evidencia que las cargas factoriales estandarizadas presentan valores superiores a 0.70, además, la probabilidad asociada al estadístico de prueba es significativo (***) ya que todos los valores son inferiores a 0,05. Las correlaciones cuadradas múltiples (R2) de cada componente es igual o superior a 0,50 .

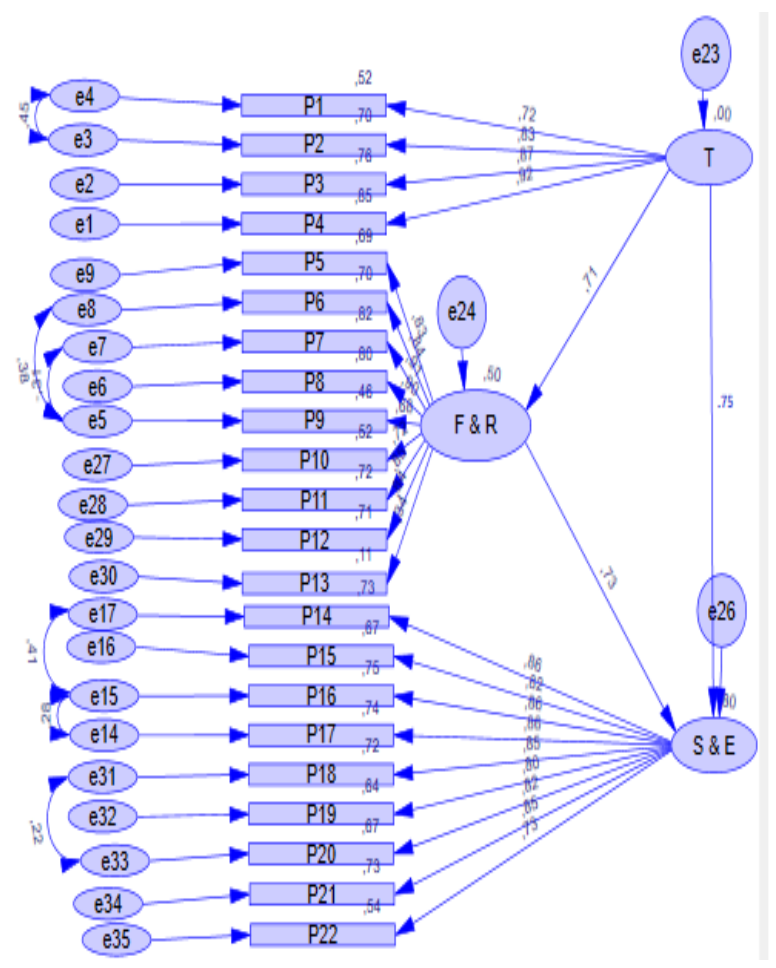

Gráfico 2 Parámetros estimados estandarizados del análisis factorial confirmatorio del modelo SERVQUAL

Fuente: Elaboración propia. 
Al evaluar los índices, y al comparar el modelo inicial y estimado, todos mejoraron y se ajustaron bien a los datos, permitiendo estimar un modelo aceptable.

Como se puede observar en la Tabla 7 Índice de bondad de ajuste, las medidas de ajuste incremental NFI $(0,888)$, IFI $(0,952)$, TLI $(0,941)$ y CFI $(0,951)$, presentan valores iguales o superiores a 0.90 que es valor mínimo requerido. El índice basado en las covarianzas del modelo RMSEA se encuentra por debajo de 0,05 , lo que indica un buen ajuste.

Tabla 6 Índices de bondad de ajuste

\begin{tabular}{lrrrrrrrrr}
\hline & \multicolumn{1}{c}{ Modelo de Ajuste Absoluto } & \multicolumn{6}{c}{ Medidas de Ajuste Incremental } \\
\hline Modelos & $\begin{array}{c}\text { Chi- } \\
\text { cuadrado }\end{array}$ & gl & RMSEA & CFI & IFI & TLI & NFI & GFI \\
Modelo Inicial & 2231,2 & 872 & 0,198 & 0,534 & 0,532 & 0,641 & 0,635 & 0,621 \\
$\begin{array}{l}\text { Modelo } \\
\text { Modificado }\end{array}$ & 321,6 & 192 & 0,050 & 0,951 & 0,952 & 0,941 & 0,888 & 0,775 \\
& & & & & & & & & \\
\hline
\end{tabular}

Fuente: Elaboración propia

\section{Discusión.}

La validez nomológica de una herramienta como SERVQUAL, al no tener un criterio de referencia en la amazonía ecuatoriana, se hace necesaria, considerando a la metodología SERQUAL como un estándar de validación y dentro de ella los factores motivo de esta investigación, por lo tanto, aquí se destaca la necesidad de precisar los términos esenciales de la medición, exclusivamente para alojamiento de la amazonia ecuiatoriana, como punto de partida para incorporar criterios confirmatorios en futuras investigaciones.

Para el área médica, recurrentemente las variables de interés son de tipo no observable, en el imaginario humano abstracciones como fenómenos que permiten comunicar, diferenciar, definir o generalizar una «variable latente»o «constructo no observable», es necesario hacerlo de forma indirecta por medio de «indicadores observables» (Joan Manuel. BatistaFogueta, 2004), así mismo es necesario orientar la evaluación en el turismo, postulado que 
coincide con el criterio de esta investigación, al reconocer al servicio de alojamiento o al proceso mismo de servucción como intangible que necesita ser medido.

Al someter a los datos obtenidos al análisis factorial confirmatorio, se detallan algunos pormenores que no son percibidos en una primera impresión, pero luego del tratamiento, se los identifica de una manera mucho mas clara, obteniendo rangos que llama la atención al momento de realizar el análisis e interpretación de resultados, no así con los demás que muestran fiabilidad en su uso.

El sector turístico del Ecuador cuenta con una planificación en la que se encuentra un acápite importante de publicidad y promoción, que deberá considerar la discriminación de los contenidos que estarán mostrados en su oferta, por lo tanto, los resultado de todas las investigaciones pertinentes a la actividad turísitica tienen un componente importante para ser socializado no solo con los prestadores de servicios sino con los actores políticos para que sean difundidos de la mejor manera y con esa información orientar las decisiones de todo el sector.

La ciudad de Tena al tener en su plan de desarrollo un área especial de turismo, requiere de investigaciones que permitan orientar a la SUERESTRUCTURA turística el verdadero accionar en territorio, considerando herramientas que viabilicen en la parte operativa con las necesidades de los clientes y la formación de los prestadores de servicios con capacitación efectiva en los temas que se requieran de manera puntual; al hablar de percepción y expectativa la realidad de las personas que pretenden visitar versus a los que ya lo han hecho, este trabajo orienta, con base teórica y metodológica la formalización del turismo y al mismo tiempo su efectiva oferta de servicios.

\section{Conclusiones.}

- Al aplicar el modelo SERVQUAL a los establecimientos de alojamiento de la ciudad de Tena se encontró que las expectativas, medidas por los aspectos tangibles, fiabilidad, capacidad de respuesta, seguridad y empatía son considerados importantes para el cliente, ya que el puntaje medio obtenido de la encuesta aplicada a estos se ubicó en el rango de 6.01 y 6.78, que corresponde una alta expectativa. 
- Los estadísticos descriptivos de las expectativas, presentaron un puntaje medio entre 4.87 y 4.91 los que corresponden a la dimensión aspectos tangibles y una pregunta que forma parte de la dimensión capacidad de respuesta, indicando que son los aspectos menos importantes para los encuestados.

- Las diferencias entre las percepciones y expectativas, indicaron que lo percibido fue menor a lo esperado por los clientes. De ello se obtuvo que los aspectos que mejor desempeño obtuvieron fueron las dimensiones aspectos tangibles, capacidad de respuesta y empatía.

- El análisis factorial comprobatorio de los 22 ítems y 3 dimensiones o variables no observables, agrupadas de la siguiente manera: para la Dimensión, Tangibilidad (T), Fiabilidad, Capacidad de Respuesta (F \& R) y Seguridad y Empatía (S y E), mostró que todos 20 ítems explicaban el modelo y cumplieron con las especificaciones (fiabilidad individual >0,50) y 2 items no cumplieron con las especificaciones (fiabilidad individual $>0,50$ )

\section{Referencias bibliográficas.}

Arciniegas, J., \& Mejías, A. (2017). Percepción de la calidad de los servicios prestados por la Universidad Militar Nueva Granada con base en la escala Servquialing, con análisis factorial y análisis de regresión múltiple. Comunicación: Revista de Investigación en Comunicación y Desarrollo, 8(1), 26 - 36.

Arias, F. (2012). El proyecto de Investigación (Sexta ed.). Caracas: Episteme.

Arias, F. (2016). El Proyecto de Investigación. Caracas: Epísteme.

Barreiro, A. (2015). Plan Turístico Comunitario en el Valle de Tumbaco para el crecimiento de la actividad turística. Quito: Instituto Tecnológico Cordillera.

Crespo, J. (2019). Perfil del turista ecológico, aspectos sociodemográficos, expectativas y actividades del ecoturista en España. Revista Interamericana de Ambiente, 15(2), 192 - 201.

GAD Municipal de Tena. (02 de Septiembre de 2020). Datos Geográficos Tena. Obtenido de Recuperado de: https://tena.gob.ec/index.php/tena/canton-tena 
Guamangallo, E., Masapanta, D., \& Nasimba, C. y. (2019). Performance of the Q distintive in the Cantón Mejía in Ecuador. Revista Científica Electrónica de Ciencias Gerenciales, 4(44), 47 - 61.

Hernández-Sampieri, R., Fernández-Collado, C., Baptista-Lucio, P., a, a, a, . . a a. (2014). Metodología de la Investigación (Sexta ed.). México: Mc Graw Hill.

JM. BATISTA-FOGUET. COEDERS G., A. M. (2001). sing structural equation mo-dels to evaluate the magnitude of measurement error in blood pressure. Statistics in Medicine, 20-68.

Joan Manuel. Batista-Fogueta, G. C. (2004). Análisis factorial confirmatorio. Su utilidad en la validaciónde cuestionarios relacionados con la salud. Researchgate.net, 21.

MINTUR. (2018). Principales Indicadores de Turismo. Quito: Mintur.

Morales, M., \& Fernández, M. y. (2019). Análisis del turismo rural y de la sostenibilidad de los alojamientos rurales. Revista Espacios, 40(1), 1 - 14.

Qualitur. (03 de Septiembre de 2020). Sistema de calidad turística española. Obtenido de Recuperado de:http://www.qualitur.org/masmedios/documentos/noticia127.doc

Quispe, G., \& Ayavarí, V. (2016). Medición de la satisfacción del cliente en organizaciones no lucrativas de cooperación al desarrollo. Riobamba: Universidad Nacional de Chimborazo.

Salazar, W., \& Cabrera, M. (2016). Diagnóstico de la calidad de servicio, en la atención al cliente, en la Universidad Nacional de Chimborazo - Ecuador. Industrial Data, 19(2), 13 - 20.

Ventura, J. (2017). Población o muestra; Una diferencia necesaria. Revista Cubana de Salud Pública, 43(4), 648 - 649.

Zeithalm, V. (2000). Service quality, profitability, and the economic worth of customers: What we know and what we need to learn. Journal of the Academy of Marketing Science, 28(1), 67-85.

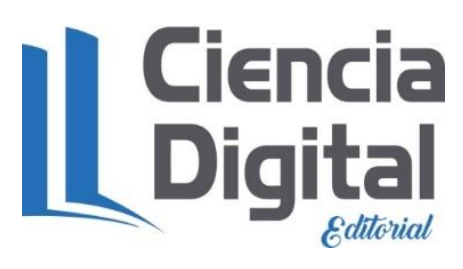




\section{PARA CITAR EL ARTÍCULO INDEXADO.}

Rivadeneyra Olalla, G. P., Ruiz Mármol, E. E., Manosalvas Vaca, C. A., \& Toalombo Díaz, Álvaro S. (2020). Análisis factorial comprobatorio de la herramienta SERVQUAL en alojamiento. Amazonia Ecuador. Explorador Digital, 4(4), 90-106. https://doi.org/10.33262/exploradordigital.v4i4.1428

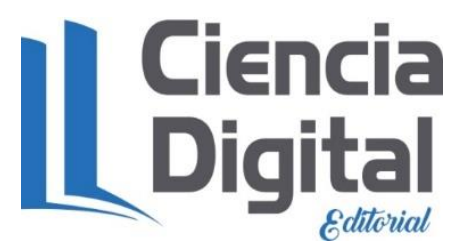

El artículo que se publica es de exclusiva responsabilidad de los autores y no necesariamente reflejan el pensamiento de la Revista Explorador Digital.

El artículo queda en propiedad de la revista y, por tanto, su publicación parcial y/o total en otro medio tiene que ser autorizado por el director de la Revista Explorador Digital.
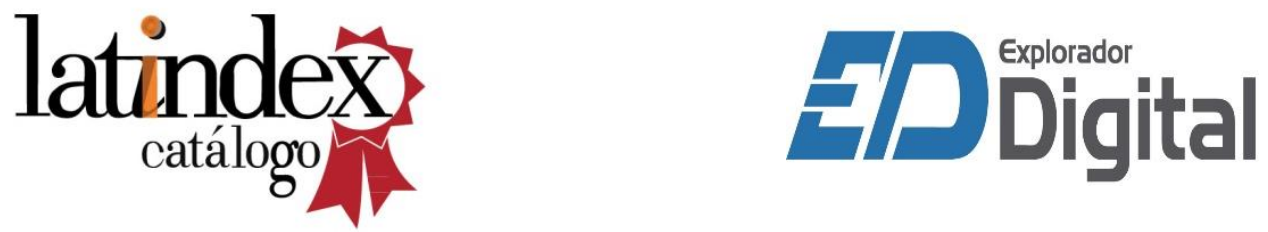